\title{
Vesicouterine Fistula in Burkina Faso: Report of 36 Cases in a Multicentric Study
}

\author{
Boureima Ouedraogo, ${ }^{1}$ Brahima Kirakoya, ${ }^{2}$ Moussa Kabore, ${ }^{2}$ Adama Millogo, ${ }^{2}$ \\ Adama Ouattara, ${ }^{3}$ Fasnewinde Aristide Kaboré ${ }^{\infty 2}$ \\ ${ }^{1}$ Urology Department University Hospital of Tingandogo, Burkina Faso ${ }^{2}$ Department of Urology and Andrology, University Hospital Yalgado Ouédraogo of Ouagadougou, \\ Burkina Faso ${ }^{3}$ Urology Department University Hospital Souro Sanou of Bobo-Dioulasso, Burkina Faso
}

\section{Abstract}

Objective To report etiological and therapeutic features of vesicouterine fistulas (VUF) in Burkina Faso.

Patients and Methods We performed a retrospective, descriptive, and multicentric study based on the medical records of women treated for VUF from January 2010 to December 2016.

Results VUF accounted for 7.2\% (36/497) of urogenital fistulas managed during the study period. The median age of the 36 patients was 35 years (interquartile range $=27$ to 37.5 years) with values ranging from 16 years to 64 years old. Among VUF, obstetric fistula accounted for 26 cases (26/36) versus 10 cases (10/36) of iatrogenic fistula. Obstetric VUF were consecutive to emergency Caesarean section $(n=16)$ and vaginal delivery $(n=10)$ after prolonged obstructed labor. The 10 cases of iatrogenic VUF were subsequent to prelabour Caesarean section. The main circumstance of VUF occurrence was Caesarean section (26/36). In 10 cases (10/36), VUF was associated with a vesicovaginal fistula. Thirty days after the removal of the catheter, the success rate fell from $89 \%$ to $80.6 \%$.

Conclusion VUF is rare but its frequency is not negligible in our context. The main circumstance of occurrence remains Caesarean section. The best treatment remains prevention

\section{Introduction}

Vesicouterine fistula (VUF) is an abnormal communication between the posterior wall of the bladder and the anterior wall of the uterus[1,2]. It is an uncommon condition compared with vesicovaginal fistula and mainly affects young women in their thirties $[1,3]$. It is such a rare phenomenon that the literature consists mainly of cases reports and case series. VUF accounts for $1 \%$ to $4 \%$ of all urogenital fistulas, with an increasing trend reported in the literature $[1,4,5]$. In 2014, Kaboré et al. performed a prospective cohort study of 170 patients in Burkina Faso managed for urogenital fistula (UGF) and reported a frequency of $8.2 \%$ for VUF[6].

VUF usually presents with a classic triad of symptoms described by Youssef: cyclical hematuria, amenorrhea, and urinary continence[7], although patients with VUF may sometimes present with a permanent urine leakage through the genital tract. Like vesicovaginal fistulas, VUFs have a devastating psychosocial and economic impact on the women who experience them.

The most common etiology of VUF is iatrogenic through pelvic surgery. Caesarean section is the most common

\section{Key Words}

Vesicouterine fistula, Caesarean, bladder, uterus, Burkina Faso

\section{Competing Interests}

None declared.

\section{Article Information}

Received on February 13, 2021

Accepted on May 13, 2021

Soc Int Urol J. 2021;2(4):210-215

DOI: 10.48083/NFM02987 


\section{Abbreviations}

IQR interquartile range

UGF urogenital fistula

VUF vesicouterine fistula

cause reported in the literature, accounting for approximately $83 \%$ to $93 \%$ of cases of VUF[8]. In Burkina Faso, Sombié et al. reported an increase in Caesarean section rate from $0.48 \%$ in 2000 to $2.1 \%$ in 2014 [9]. This trend of increasing Caesarean section rates will likely be accompanied by an increase in the incidence of VUF. Treatment of VUF can be conservative or surgical. However, conservative treatment has shown a low success rate in contrast to surgical treatment which provides good results[10].

The aim of the present study was to analyze the etiological and therapeutic aspects of VUF in Burkina Faso through a multicentric study.

\section{Materials and Methods}

\section{Study design and period}

We conducted a multicentric retrospective study over a 7-year period (from January 1, 2010, to December 31, 2016).

\section{Study site and population}

Our study population consisted of all patients managed for urogenital fistula at 7 referral centers for the treatment of urogenital fistulas in Burkina Faso: University Hospital Yalgado Ouédraogo of Ouagadougou, Regional Hospital of Fada N'gourma, Regional Hospital of Dori, Saint Camille Hospital in Ouagadougou, New PolyClinic of the Center in Ouagadougou, Medical Center with Surgical Antenna in Boromo, and Medical Center with Surgical Antenna of Schipphra in Ouagadougou. We analyzed the medical records of all patients managed for VUF.

\section{Definition of variables}

The following variables were studied for patients with VUF: age, parity, female genital mutilation (Yes or No), history of prior fistula repair (Yes or No), duration of labor, time between fistula onset and the surgical repair, fistula size, Jóźwik's classification, etiological factors (obstetric and iatrogenic), duration of bladder catheterization, and postoperative results (success or failure). Etiological factors were divided into 2 groups: iatrogenic fistulas following prelabour Caesarean section, and obstetrical fistulas following vaginal delivery or emergency Caesarean section performed too late. We used the classification of VUF into 3 types proposed by Jóźwik[11]: type I presents with amenorrhea and cyclic menouria without urinary incontinence; type
II presents with cyclic menouria, but has regular menses and urinary incontinence; and type III presents with only urinary incontinence, without menouria and with normal menses. Data were collected on an individual and anonymous data sheet.

This study was performed in accordance with the ethical standards laid down in the Declaration of Helsinki.

\section{Analysis and measures}

Data were analyzed using the SPSS software, version 21.0. The categorical variables were analyzed through the study of frequencies. The continuous variables were analyzed through the study of median and interquartile range (IQR), minimum and maximum. We defined a successful surgical repair outcome at one month as a complete closure of the defect attested by a methylene blue test and without urinary incontinence. The Shapiro-Wilk test was used to assess the normality for continuous variables. The continuous variables do not follow a normal distribution in the sample of 36 patients. We therefore used the medians.

\section{Results}

During the study period, 497 cases of urogenital fistula were managed in the 7 centers. VUF accounted for $7.2 \%(36 / 497)$ of all urogenital fistulas. The annual frequency of VUF was 5.14. The annual distribution of VUF is presented in Figure 1. The median age of the 36 patients was 35 years (IQR $=27$ to 37.5 years), with values ranging from 16 years to 64 years old.

According to Jóźwik's classification, 31 cases (31/36) were type II. In 10 cases (10/36), VUF was associated with a vesicovaginal fistula. Obstetric fistula was noted in 26 cases (26/36) versus 10 cases (10/36) for iatrogenic fistula. Among patients with obstetric fistulas, 5 patients $(05 / 26)$ gave birth at home without medical assistance. The main circumstance of VUF occurrence was Caesarean section (26/36). The median duration of VUF was 125.8 months ( IQR $=108$ to 136.5 ), with values ranging from 24 months to 144 months. Patient and VUF characteristics are summarized in Table 1.

The management of VUF was surgical in all patients. The transperitoneal route was used in 30 cases (30/36). A vaginal hysterectomy was performed in 6 cases $(06 / 36)$. The 10 cases of associated vesicovaginal fistula were repaired during the same surgical procedure. Urine drainage was performed by systematic transurethral bladder catheterization in all cases after the surgery. The median duration of bladder catheterization was 14 days ( $\mathrm{IQR}=12$ to 15.8 ), with values ranging from 10 to 21 days. Four patients $(4 / 36)$ had postoperative complications: urinary tract infection in 3 patients, and hematuria in 1 patient. All patients were evaluated on 


\section{FIGURE 1.}

Annual distribution vesicouterine fistulas $(n=36)$

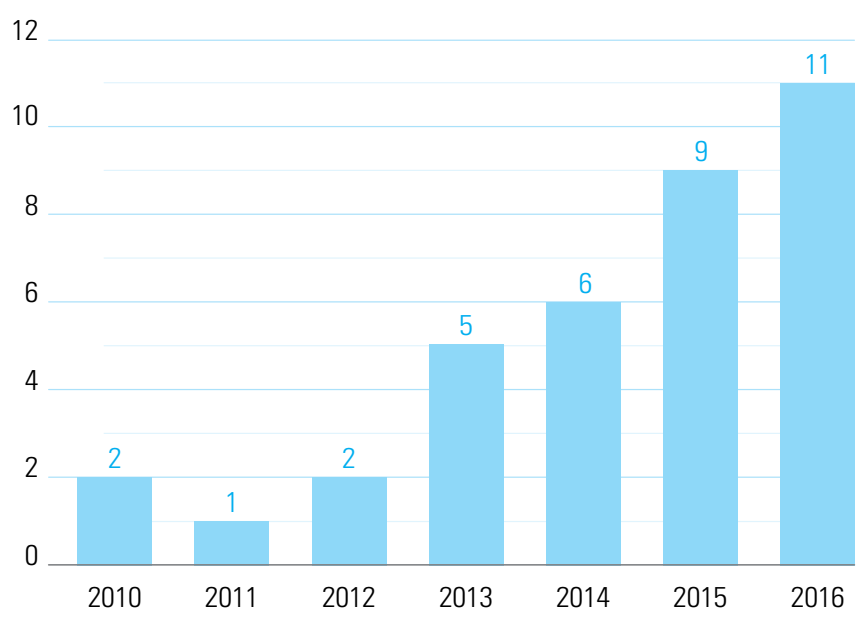

the day of bladder catheter removal and 1 month after.

\section{Discussion}

VUF is a very rare condition that has been estimated to account for $1 \%$ to $4 \%$ of all genitourinary fistulas $[1,4,5,12]$. However, recent studies have shown an increase in the prevalence of VUF. In a study of 272 women with obstetric fistula, Egziabher et al. reported $23 \%$ had VUF[13]. In 2020, Richter et al., describing the characteristics of genitourinary fistulas in Kigali, Rwanda, reported 185 (29\%) cases of vesicouterine/ vesicocervical fistula[14]. In our series, we report 36 cases of VUF, accounting for $7.2 \%$ of all genitourinary fistulas. To our knowledge it is one of the largest case series published to date. Indeed, these 7 centers are referral centers for the treatment of urogenital fistulas in Burkina Faso. Most of the published literature consists of cases reports and case series. Benchekroun et al. reported 30 cases over a 25 -year period[15]. HadziDjokic et al. reported 14 cases over a 37-year period[16].

Two main factors may explain the increasing trend in the prevalence of VUF in low-resource countries: the trend of increasing Caesarean section rate (with nonspecialist staff sometimes deciding on the procedure without strong medical indication), and the high number of unassisted deliveries[5,17]. According to Sombié et al., the rate of Caesarean section delivery in Burkina Faso increased from $0.48 \%$ in 2000 to $2.14 \%$ in 2014 [9]. VUFs most commonly occur following lowsegment Caesarean section $[1,2,10,11,18,19]$. In a review including reports from 1986 to 1997, 83\% of VUF were associated with Caesarean section[20]. In the present study, most VUF (26/36) occurred following Caesarean section. The mechanism by which communication occurs between bladder and uterus may be a bladder injury during incision of the lower segment of the uterus, during vesicouterine detachment or by uterine rupture affecting the bladder. Excessive intraoperative bleeding may also lead to injury from attempts to achieve hemostasis [2,5].

VUF has been described following dystocic vaginal deliveries. It occurs in patients with a scarred uterus or after instrumental extraction $[1,3,21,22]$. The posterior wall of the bladder becomes progressively devitalized due to changes in the vascular network at the scar of the first operation. A dehiscence of the uterine scar and simultaneous injury of the bladder wall at the vesicouterine interface may occur with the thinning of the lower segment during labor, leading to the fistula[2,5]. In the present study, dystocia was the second most common cause of VUF. Drissi et al.[1] and Hodonou et al.[3] made the same observation. None of the patients in our study had a scarred uterus, and no instrumental extraction was noted. Deliveries were often unassisted, and 5 patients delivered at home. Other causes of VUF reported in the literature are gynecological (migration of an intrauterine device, degenerated or adherent myoma of the bladder) or congenital $[1,2,7]$. We did not find these causes in our study.

The purpose of VUF treatment is to suppress communication between uterus and bladder. Two therapeutic approaches are possible: conservative management and surgical management. Conservative management combines indwelling bladder catheterization with hormonal treatment that suppresses menstruation for 3 to 6 months[2,23,24]. Spontaneous closure of small fistulas seen at an early stage has been reported[2,23], providing some justification for conservative management. However, results are generally disappointing, with Jóźwik et al. estimating the success rate to be about 5\%[24]. In our series, no patients were conservatively managed. We agree with Sylla et al. that large or late-onset VUF should receive surgical treatment[25]. DiMarco et al. in a series of 8 cases of VUF undertook conservative management of 2 patients, but this was not successful, and surgical intervention was eventually required[10]. This consists of the excision of the fistulous pathway and necrotic tissue and separate suturing of the bladder and uterus by either the extraperitoneal or transperitoneal route[1]. Bladder catheterization is maintained for about 2 weeks $[5,21]$. Hysterectomy is an option if no further pregnancy is desired. Vaginal hysterectomy was performed in 6 cases $(16.7 \%)$ in our series. The laparoscopic approach is reported in the literature with similar results to open surgery $[4,25]$. VUF repair was performed by open surgery in our study. Our results were similar to those 


\section{TABLE 1.}

Patient characteristics and vesicouterine fistula characteristics $(n=36)$

\begin{tabular}{|c|c|c|c|}
\hline & Median & $\operatorname{IOR}($ Min, Max) & Frequency \\
\hline Age, years & 35 & $27-37.5(16,64)$ & \\
\hline Parity & 4.1 & $2.6-5.6(1,11)$ & \\
\hline \multicolumn{4}{|l|}{ Genital mutilation } \\
\hline - No & & & 30 \\
\hline - Yes & & & 06 \\
\hline \multicolumn{4}{|l|}{ Etiology } \\
\hline \multicolumn{4}{|l|}{ Obstetric } \\
\hline - Vaginal delivery & & & 10 \\
\hline - Emergency Caesarean section performed too late & & & 16 \\
\hline latrogenic (prelabour Caesarean section) & & & 10 \\
\hline \multicolumn{4}{|l|}{ Jóźwik's classification } \\
\hline - Type I & & & 02 \\
\hline - Type II & & & 31 \\
\hline - Type III & & & 03 \\
\hline
\end{tabular}

Duration of fistula, months

$<24$

\begin{tabular}{l|c|c|}
\hline Duration of labor, hours & 19.7 & $17.6-22.6(01,24)$ \\
\hline Fistula size, cm & 02 & $1.4-2.8(01,04)$ \\
\hline
\end{tabular}

\section{Prior repair of VUF}

$$
\text { - Yes }
$$

reported in the literature, with a successful closure rate of $88.9 \% 1$ week after indwelling catheter removal. This excellent early result decreased to $80.6 \%$ after 1 month. This finding suggests the need for monitoring of patients undergoing VUF or, more generally, urogenital fistula surgery. Poor tissue vascularization and fibrosis are responsible for poor wound healing after surgery[26]. In addition, special attention has to be paid to the complete excision of perifistular necrotic tissue during surgery to allow better healing of tissues already weakened by ischemia. The postoperative failure rate reflects the difficulty of closing some urogenital fistula, sometimes after several re-interventions[11,26]. In the present study, 17 patients (17/36) had a prior surgical repair of their VUF.

Caesarean section must be medically indicated, and more obstetricians and specialized nurses may be required to perform this procedure more proficiently. Meda et al. showed that in Burkina Faso, only 39.2\% of Caesarean sections are performed by obstetricians and $28.7 \%$ by nurses specialized in surgery[27]. Every effort 
must be made to prevent urogenital fistula, in particular by ensuring all women have access to prenatal care and to skilled medical assistance in delivery.

The limitations of this study lie in its retrospective nature, which means that some data were not available. Also, the postoperative follow-up of our patients was limited to one month. This study did not take into account data from all the urogenital fistula centers in Burkina Faso.

\section{References}

1. Drissi M, Karmouni T, Tazi K, El Khader K, Koutani A, Ibn Attya A, et al. Vesico-uterine fistulas: a 17-year experience. Prog Urol.2008;18:173176. doi: 10.1016/j.purol.2007.12.011.

2. Karaltı MO, Tınar S, Öztürk NT, Öztekin DC, Öztürk DC. A case with vesicouterine fistula: mini review. Arch Gynecol Obstet.2012;285:667-70.

3. Hodonou R, Hounnasso P, Biaou 0, Akpo C. Vesico-uterine fistula: about 15 cases at the Cotonou University Urology Clinic. Prog Urol.2002;12:641-645.

4. Alamoudi OJ, AITheneyan MA, Aldhaam N, Moazin M. Early robotic repair of vesicouterine fistula : a case report and literature review. Urol Case Rep.2017;11:76-78.

5. Sefrioui O, Benabbes Taarji H, Azyez M, Aboulfalah A, El Karroumi M, Matar N, et al. Vesico-uterine fistulas of obstetrical origin. About 3 cases. Ann Urol (Paris).2002;36:376-380.

6. Kaboré FA, Kambou T, Ouattara A, Zango B, Yaméogo C, Kirakoya $B$, et al. Epidemiological, etiological aspects and psychosocial impact of urogenital fistula in a cohort of 170 consecutive patients managed in three centers in Burkina Faso from 2010 to 2012. Prog Urol.2014;24:526-532.

7. Youssef AF. The uterine isthmus and its sphincter mechanism, a radiographic study. I. The uterine isthmus under normal conditions. Am J Obstet Gynecol.1958;75:1305-1319. DOl:https://doi. org/10.1016/0002-9378(58)90721-X

8. Bhattacharjee S, Kohli UA, Sood A, Tripathy S, Gupta M. Vesicouterine fistula: Youssef's syndrome. Med J Armed Forces India.2015;71:175-177.

9. Sombié I, Meda ZC, Savadogo G, Sanou G, Dadjoari M. Trends in the rate and place of cesarean section deliveries in Burkina Faso. Santé Publique.2017;29:133.

10. DiMarco CS, DiMarco DS, Klingele CJ, Gebhart JB. Vesicouterine fistula : a review of eight cases. Int Urogynecol J.2006;17:395-399.

\section{Conclusion}

Globally, VUF is rare, but it presents a significant challenge in some areas. It occurs mainly after Caesarean section. The results of surgical treatment are good, but the best treatment remains prevention through pregnancy monitoring and delivery in medical facilities.

\section{Acknowledgments}

The authors thank Mr Boyo Pare for English language revision.

11. Józwik M, Józwik M. Clinical classification of vesicouterine fistula. Int J Gynecol Obstet.2000;70:353-357.

12. Saeed S, Nawaz N, Murtaza B, Mahmood A. Urogenital fistula in females - a four years experience. Pak Armed Forces Med J.2016;66:361-366.

13. Egziabher TG, Eugene N, Ben K, Fredrick K: Obstetric fistula management and predictors of successful closure among women attending a public tertiary hospital in Rwanda: a retrospective review of records. BMC Res Notes.2015;8:774. doi: 10.1186/ s13104-015-1771-y.

14. Richter LA, Lee $H$, Nishimwe $A$, Niteka LC, Kielb SJ. Characteristics of genitourinary fistula in Kigali, Rwanda; 5-year trends. Urology.2021; 150:165-169. doi: 10.1016/j.urology.2020.05.077. Epub 2020 Jun 24.

15. Benchekroun A, El Alj HA, El Sayegh H, Lachkar A, Nouini Y, Benslimane $\mathrm{L}$, et al. Les fistules vésico-vaginales : à propos de 1050 cas. Ann Urol.2003;37:194-198.

16. Hadzi-Djokic JB, Pejcic TP, Colovic VC. Vesico-uterine fistula: report of 14 cases. BJU Int.2007;100:1361-1363.

17. Ouédraogo CM, Ouédraogo A, Ouattara A, Lankoandé J. The practice of cesarean section in a district hospital in Ouagadougou Epidemiological, clinical and prognostic aspects for 3,381 cases. Med Sante Trop.2015;25:194-199.

18. Józwik M, Józwik M, Łotocki W. Vesicouterine fistula — an analysis of 24 cases from Poland. Int J Gynecol Obstet.1997;57:169-172.

19. Birge 0, Ozbey EG, Erkan MM, Arslan D, Kayar I. Youssef's syndrome following Cesarean section. Case Rep Obstet Gynecol.2015;2015:1-4.

20. Yip SK, Leung TY. Vesicouterine fistula: an updated review. Int Urogynecol J Pelvic Floor Dysfunct.1998;9:252-256. doi: 10.1007/ BF01901500. 
21. Goyal LD. Vesicouterine fistula following vaginal birth after Caesarean section in a multiparous woman-a rare case report and review of literature. ARC Journal of Gynecology and Obstetrics.2016;1:1-2. http://dx.doi.org/10.20431/2456-0561.0103001

22. Nouira Y, Feki W, Rhouma SB, Salah IB, Horchani A. Vesicouterine fistula as a complication of forceps delivery : a case report. Int Urogynecol J Pelvic Floor Dysfunct.2005;16:512-514.

23. Bastakoti R, Saha R. Conservative management of uterovesical fistula following primary Caesarean section. Journal of Kathmandu Medical College.2013;2:211-113.

24. Józwik M, Józwik M. Spontaneous closure of vesicouterine fistula. Account for effective hormonal treatment. Urol Int.1999;62:183-187.
25. Sylla C, Fall PA, Diallo AB, Ndoye AK, Ba M, Gueye SM, et al. Vesicouterine fistulas: about 5 cases. Prog Urol.2000;634-637.

26. Sjøveian S, Vangen S, Mukwege D, Onsrud M. Surgical outcome of obstetric fistula: a retrospective analysis of 595 patients. Acta Obstet Gynecol Scand.2011;90:753-760.

27. Meda IB, Millogo T, Baguiya A, Ouédraogo/Nikiema L, Coulibaly A, Kouanda S. Rate of and factors associated with indications for cesarean deliveries: results of a national review in Burkina Faso. Int $\mathrm{J}$ Gynaecol Obstet.2016;135(Suppl 1):S51-7. 